\title{
ALGEBRAIC LIMIT CYCLES BIFURCATING FROM ALGEBRAIC OVALS OF QUADRATIC CENTERS
}

\author{
JAUME LLIBRE ${ }^{1}$ AND YUN TIAN ${ }^{2}$
}

\begin{abstract}
In the integrability of polynomial differential systems it is well known that the invariant algebraic curves play a relevant role. Here we will see that also can play an important role with respect to limit cycles.

We show that there exits only one family of quadratic polynomial systems with an algebraic periodic orbit of degree 4 surrounding a center satisfying that an algebraic limit cycle of degree 4 can bifurcate from the period annulus of the mentioned center under quadratic perturbations.
\end{abstract}

\section{Introduction AND STATEMENT of RESUlts}

We consider polynomial differential systems in $\mathbb{R}^{2}$ defined by

$$
\begin{aligned}
& \dot{x}=P(x, y), \\
& \dot{y}=Q(x, y),
\end{aligned}
$$

where $P, Q$ are polynomials with real coefficients such that the maximum degree of $P$ and $Q$ is at most 2. These differential systems are called simply quadratic systems. The dot denotes derivative with respect to the independent variable $t$, which as usual we call the time.

A system (1) has associated the quadratic polynomial vector field

$$
\mathcal{X}=P(x, y) \frac{\partial}{\partial x}+Q(x, y) \frac{\partial}{\partial y} .
$$

Sometimes we refer to $\mathcal{X}$ as a quadratic system. There are more than one thousand papers dedicated to study the quadratic systems, see for instance the surveys of Coppel [7] and of Chicone and Tian [6], and the book of Ye [24].

A singular point $p$ of a quadratic system is a center if it has a neighborhood $U$ such that every orbit in $U \backslash\{p\}$ is periodic. A center of a

\footnotetext{
Key words and phrases. quadratic systems, quadratic vector fields, quadratic center, periodic orbit, limit cycle, bifurcation from center, cyclicity of the period annulus. 2010 Mathematics Subject Classification: 34C05.
} 
quadratic system is called a quadratic center. The connected set formed by all periodic orbits surrounding a center $p$ which has as inner boundary the point $p$ is called the period annulus of the center $p$.

A periodic orbit isolated in the set of all periodic orbits of a quadratic system (1) is called a limit cycle. Questions related with the limit cycles, also for quadratic systems the more easier polynomial differential systems after the linear ones, always are hard to solve, see for instance the book of Christopher and Li [4].

It is known that quadratic differential systems having a center have no limit cycles (see [21] and/or [20]). But by perturbing the periodic orbits of a center of a quadratic system inside the class of quadratic systems some limit cycles could arise.

The sharp upper bound of the number of limit cycles which could arise from the periodic orbits of a period annulus $A$ of a quadratic center when it is perturbed within the class of all quadratic systems is called the cyclicity of the period annulus $A$. A survey about this cyclicity can be found in [4] and in the references quoted therein. In general such a cyclicity is not easy to compute.

Let $f=f(x, y)$ be a real polynomial in the variables $x$ and $y$. The algebraic curve $f=0$ is invariant for the quadratic system $\mathcal{X}$ if $\mathcal{X} f=k f$ for some polynomial $k=k(x, y)$. Since $\mathcal{X} f=<(P, Q),\left(f_{x}, f_{y}\right)>=0$ on the points $(x, y)$ of $f=0$, where $<\cdot, \cdot>$ denotes the inner product of $\mathbb{R}^{2}$, the vector field $\mathcal{X}$ is tangent to the curve $f=0$, so the curve $f=0$ is formed by orbits of $\mathcal{X}$. This explains the given name of invariant algebraic curve to $f=0$.

If a periodic orbit or a limit cycle is contained in an irreducible invariant algebraic curve $f=0$ in $\mathbb{R}[x, y]$ (the ring of all real polynomials in the two variables $x$ and $y$ ) of degree $d$, then we say that we have an algebraic periodic orbit of degree $d$ or an algebraic limit cycle of degree $d$ respectively.

In 1958 started to be studied the algebraic limit cycles of the quadratic systems. At this moment for the quadratic systems we know 7 different families of algebraic limit cycles, 1 of degree 2 (see [19] and [24]), 4 of degree 4 (see [23], [12], [3] and [1]), 1 of degree 5, and 1 of degree 6 (see [5]). Quadratic systems has no algebraic limit cycles of degree 3, see Evdokimenco [9, 10, 11], for shorter proofs see [2, 3]. It is an open question to know if quadratic systems can have algebraic limit cycles of degree $>6$. More information about the algebraic limit cycles of quadratic systems can be found in [14] and [15]. 
Here we are interested in algebraic limit cycles of degree 4 which bifurcate from some algebraic periodic orbits of degree 4 contained in a period annulus of a quadratic center. This problem for algebraic periodic orbits of degree 2 has been studied in [13].

Yablonskii [23] in 1966 found the first class of algebraic limit cycles of degree 4 within the quadratic systems. Filiptsov [12] in 1973 found the second class. Two new classes of algebraic limit cycles of degree 4 has been found in 2001, see [1] and [3]. Moreover, these authors proved that there are no other classes of quadratic systems with algebraic limit cycles of degree 4 .

Theorem 1. Assume that we have a quadratic center whose period annulus has an algebraic periodic orbit of degree 4, and can further produce an algebraic limit cycle of degree 4 under proper quadratic perturbations. Then the following statements hold.

(a) There exists a real affine change of variables and a rescaling of the time such that the quadratic system with such a center can be written as

$$
\begin{aligned}
& \dot{x}=-(a+b) y+4 x y, \\
& \dot{y}=a b(a+b) x+\left(4 a b-3 / 2(a+b)^{2}\right) x^{2}+8 y^{2},
\end{aligned}
$$

with $a \neq b, a b>0$ and $(3 a-b)(a-3 b)<0$. For such a quadratic system the algebraic curve $x^{2}(x-a)(x-b)+y^{2}=0$ contains an algebraic periodic orbit of degree 4 . The qualitative phase portraits in the Poincaré disc of these centers are given in Figure 1.

(b) When we perturb a quadratic center of (2), inside the class of all quadratic systems there is at least one 1-parameter family of quadratic systems having a limit cycle bifurcating from the algebraic periodic orbit of the curve $x^{2}(x-a)(x-b)+y^{2}=0$. Moreover this family is formed by algebraic limit cycles of degree 4 .

For more details on the Poincaré disc, canonical regions and separatrices see $[8,16,17,18]$.

From Theorem 1 we shall obtain easily the next result.

Corollary 2. Suppose that we have a quadratic center whose period annulus has an algebraic periodic orbit of degree 4. Then the cyclicity of that period annulus is at least 1. 


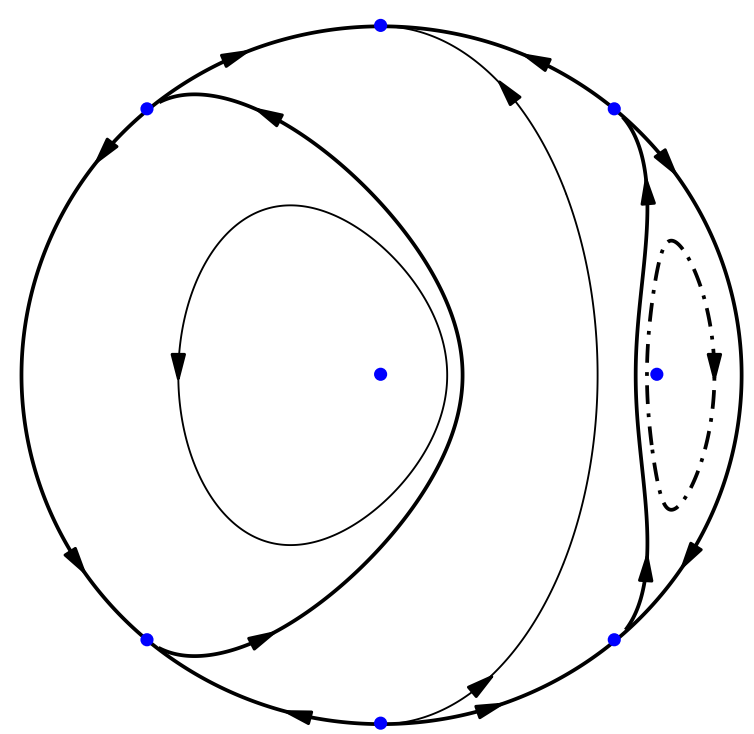

FiguRE 1. The phase portrait of system (2). It has 3 canonical regions and 16 separatrices. The dash-dot line represents the algebraic periodic orbit.

\section{Proof of Theorem 1}

Proof of statement (a) of Theorem 1. In paper [3] it is proved that there are only 4 families of algebraic limit cycles of degree 4 in quadratic systems, which are Yablonskii's system, Filiptsov's system and other two systems given in [3]. Then for any quadratic center producing algebraic limit cycles under proper quadratic perturbations, the perturbed system can be transformed into one of these four families by a suitable linear change of variables and a rescaling of the time. Then we only need to study whether there exist quadratic centers in the closure of these four families of systems in the space of all planar quadratic systems.

First we consider Filiptsov's system

$$
\begin{aligned}
& \dot{x}=6(1+a) x+2 y-6(2+a) x^{2}+12 x y, \\
& \dot{y}=15(1+a) y+3 a(1+a) x^{2}-2(9+5 a) x y+16 y^{2},
\end{aligned}
$$

with $0<a<3 / 13$, which has the irreducible invariant algebraic curve

$$
3(1+a)\left(a x^{2}+y\right)^{2}+2 y^{2}(2 y-3(1+a) x)=0 .
$$

It is known that inside any limit cycle of a quadratic system there is always a focus [24]. For system (3) the focus inside the algebraic limit 
cycle contained in the curve (4) is $E_{f}=\left(\frac{5(1+a)}{2(1-a)}, \frac{15(1+a)^{2}}{8(1-a)}\right)$. Then we need to find whether $E_{f}$ is a center point when $a=0$ or $3 / 13$. The charateristic equation at $E_{f}$ is

$$
\lambda^{2}-\frac{(13 a-3)(1+a)}{2(1-a)} \lambda+\frac{15(11 a+9)(1+a)^{2}}{1-a}=0 .
$$

If $a=0$, the roots of the above equation are $-\frac{3}{4} \pm i \frac{3}{4} \sqrt{239}$, where $i^{2}=-1$, which implies that $E_{f}$ is an elementary focus. If $a=3 / 13$, the equation has a pair of purely imaginary conjugate roots. Then $E_{f}$ is the linear center of the corresponding linearized system of (3). Furthermore, $E_{f}$ is a weak focus of system (3) because the first order Lyapunov constant $V_{1}=$ $5 / 2$ is different from zero [22]. Therefore, there do not exist quadratic centers for system (3).

Similar situation happens to another two families of systems given by

$$
\begin{aligned}
& \dot{x}=5 x+6 x^{2}+4(1+a) x y+a y^{2}, \\
& \dot{y}=x+2 y+4 x y+(2+3 a) y^{2},
\end{aligned}
$$

with $(-71+17 \sqrt{17}) / 32<a<0$, and

$$
\begin{aligned}
& \dot{x}=2\left(1+2 x-2 a x^{2}+6 x y\right), \\
& \dot{y}=8-3 a-14 a x-2 a x y-8 y^{2},
\end{aligned}
$$

with $0<a<1 / 4$. System (5) has the algebraic limit cycle represented by the oval of the curve

$$
x^{2}+x^{3}+x^{2} y+2 a x y^{2}+2 a x y^{3}+a^{2} y^{4}=0 .
$$

The singular point in the region bounded by the algebraic limit cycle is

$$
\left(\frac{12 a^{2}+34 a+5+(6 a+5) \sqrt{4 a^{2}+16 a+1}}{6(2-3 a)}, \frac{6 a+7+3 \sqrt{4 a^{2}+16 a+1}}{2(3 a-2)}\right) .
$$

In order to make this singular point be a linear center, we get $a=(-71+$ $17 \sqrt{17}) / 32$, under which this singular point is a weak focus by computing the first-order Lyapunov constant $V_{1}=17^{1 / 4}(469-115 \sqrt{17}) / 32$.

For system (6), the oval of the invariant algebraic curve

$$
1 / 4+x-x^{2}+a x^{3}+x y+x^{2} y^{2}=0,
$$

is the algebraic limit cycle, and the singular point $\left(\frac{2+\sqrt{4-7 a}}{7 a}, \frac{-8+3 \sqrt{4-7 a}}{14}\right)$ is the one contained in the interior region bounded by the algebraic limit cycle. An easy computation shows that this singular point is a weak focus when $a=1 / 4$ and becomes an elementary focus when $a=0$. 
The last system we consider is Yablonskii's system

$$
\begin{aligned}
\dot{x}= & -4 a b c x-(a+b) y+3(a+b) c x^{2}+4 x y, \\
\dot{y}= & a b(a+b) x-4 a b c y+\left(4 a b c^{2}-3 / 2(a+b)^{2}+4 a b\right) x^{2} \\
& +8(a+b) c x y+8 y^{2},
\end{aligned}
$$

with $a b c \neq 0, a \neq b, a b>0$ and $4(a-b)^{2} c^{2}+(3 a-b)(a-3 b)<0$. System (7) has two singular points, the origin and the point

$$
E_{1}=\left(\frac{2 a b(a+b)}{3 a^{2}-2 a b+3 b^{2}},-\frac{4 a^{2} b^{2} c}{3 a^{2}-2 a b+3 b^{2}}\right),
$$

and the irreducible invariant algebraic curve

$$
\left(y+c x^{2}\right)^{2}+x^{2}(x-a)(x-b)=0 .
$$

The singular point $E_{1}$ is located in the bounded region limited by the oval of the curve (9). The charateristic polynomial at $E_{1}$ is

$$
\begin{aligned}
& p_{3}(\lambda)=\lambda^{2}+a_{1} \lambda+a_{0}, \text { where } a_{1}=-\frac{4 a b(a-b)^{2} c}{3 a^{2}-2 a b+3 b^{2}}, \\
& a_{0}=-\frac{a b\left((3 a-b)(a-3 b)(a+b)^{2}+16 a b(a-b)^{2} c^{2}\right)}{3 a^{2}-2 a b+3 b^{2}} .
\end{aligned}
$$

In order to have an elementary center at the singular point $E_{1}, p_{3}(\lambda)$ should have a pair of purely imaginary conjugate roots. Then $a_{1}=0$ and $a_{0}>0$. By (10), $a_{1}=0$ implies $a b(a-b) c=0$. On the other hand, $a b \neq 0$ are obtained from $a_{0}>0$. If $a-b=0$, then the algebraic curve (9) becomes

$$
\left(y+c x^{2}\right)^{2}+x^{2}(x-a)^{2}=0,
$$

which does not have any oval. Then $a \neq b$.

Now it only remains to study the case of $c=0$. If $c=0$, from (10) we have

$$
a_{0}=-\frac{a b(3 a-b)(a-3 b)(a+b)^{2}}{3 a^{2}-2 a b+3 b^{2}}>0,
$$

when we take into account $3 a^{2}-2 a b+3 b^{2}>0$ and the conditions given with system (7). It is easy to find that if $c=0$, the origin and $E_{1}$ are linear center of the corresponding linearized systems of system (7) when $a b>0$ and $(3 a-b)(a-3 b)<0$.

When $c=0$, system (7) becomes into (2), and by (8), the singular point $E_{1}$ becomes into

$$
E_{o}=\left(\frac{2 a b(a+b)}{3 a^{2}-2 a b+3 b^{2}}, 0\right) .
$$


System (2) is integrable with the first integral

$$
H=\frac{(a+b)^{4}-16(a+b)^{3} x+32\left(3 a^{2}-2 a b+3 b^{2}\right) x^{2}-256 y^{2}}{(a+b-4 x)^{4}} .
$$

The existence of the first integral defined at the origin and $E_{o}$ forces that the origin and $E_{o}$ are centers in (2). The oval $\Gamma_{o}$ of the algebraic curve $x^{2}(x-a)(x-b)+y^{2}=0$ is an algebraic periodic orbit in the period annulus around $E_{o}$.

Proof of statement (b) of Theorem 1. We take the following perturbation for system (2)

$$
\begin{aligned}
\dot{x}= & -4 a b \varepsilon x-(a+b) y+3(a+b) \varepsilon x^{2}+4 x y, \\
\dot{y}= & a b(a+b) x-4 a b \varepsilon y+\left(4 a b \varepsilon^{2}-3 / 2(a+b)^{2}+4 a b\right) x^{2} \\
& +8(a+b) \varepsilon x y+8 y^{2},
\end{aligned}
$$

where $\varepsilon$ is a nonzero small parameter satisfying $4 \varepsilon^{2}(a-b)^{2}+(3 a-b)(a-$ $3 b)<0$. If we take $\varepsilon=c$, system (11) concides with Yablonskii's system (7). Then the perturbed system (11) has an invariant algebraic curve given by

$$
\left(y+\varepsilon x^{2}\right)^{2}+x^{2}(x-a)(x-b)=0,
$$

and one of its components is an oval $\Gamma_{\varepsilon}$. Hence, by Theorem 1 in [3], $\Gamma_{\varepsilon}$ is a limit cycle in system (11) for $\varepsilon \neq 0$ sufficiently small. Futhermore, the family of limit cycles $\Gamma_{\varepsilon}$ tends to the algebraic periodic orbit $\Gamma_{o}$ as $\varepsilon \rightarrow 0$.

Denote the oval of the curve (9) by $\Gamma_{c}$. From the above result, we see that the algebraic limit cycle $\Gamma_{c}$ of Yablonskii's system is born from the algebraic periodic orbit $\Gamma_{o}$ in the periodic annulus around the center $E_{o}$ when $c=0$. Solving the algebraic equation (9) in $y$, we can express $\Gamma_{c}$ by the following two functions

$$
y_{ \pm}(x, c)=-c x^{2} \pm \sqrt{x^{2}(x-a)(x-b)},
$$

where $x \in[\min (a, b), \max (a, b)]$. Then

$$
y_{+}(x, c)-y_{-}(x, c)=2 \sqrt{x^{2}(x-a)(x-b)},
$$

which is constant with respect to $c$. That means that if $a$ and $b$ are fixed, the algebraic limit cycle $\Gamma_{c}$ only moves up or down as $c$ becomes smaller or larger in the range

$$
0<c^{2}<c^{*}, \text { where } c^{*}=-\frac{(3 a-b)(a-3 b)}{4(a-b)^{2}},
$$

but the area of the region bounded by $\Gamma_{c}$ does not change. 
Note that system (7) is invariant under the transformation $(x, y, t, c) \rightarrow$ $(x,-y,-t,-c)$. Then we only need to show the phase portrait of system (7) with $0<c<\sqrt{c^{*}}$. See Figure 2.

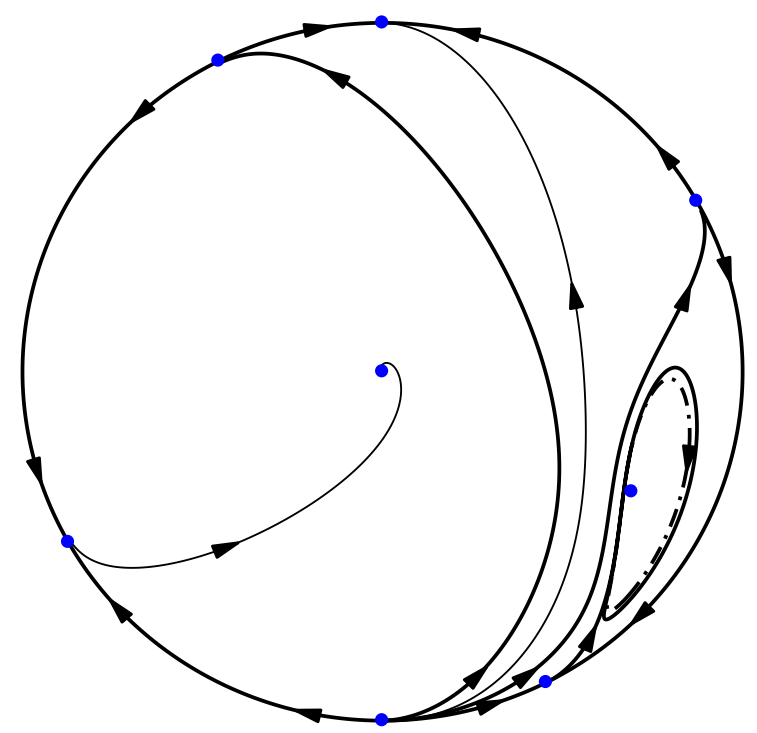

FiguRE 2. The phase portrait of Yablonskii's system (7) when $0<c<\sqrt{c^{*}}$. It has 4 canonical regions and 19 separatrices. The dash-dot line represents the algebraic limit cycle.

When $c^{2}=c^{*}$, Yablonskii's system has one more singular point

$$
E_{2}=\left(-\frac{a^{2}-6 a b+b^{2}}{2(a+b)},-\frac{(3 a-b)(a-3 b)\left(a^{2}-6 a b+b^{2}\right)}{16(a-b)^{2} c}\right) .
$$

Substituting $E_{2}$ into the equation (9), we can find that $f\left(E_{2}\right)=0$, i.e. the singular point $E_{2}$ is on the oval $\Gamma_{c}$. Then now $\Gamma_{c}$ is not a periodic orbit in system (7) now, and becomes a homoclinic loop. See Figure 3.

We can further show that $E_{2}$ is a saddle-node. The charateristic equation at $E_{2}$ is

$$
\lambda^{2}-\frac{(3 a-b)(a-3 b)}{2 c} \lambda=0
$$




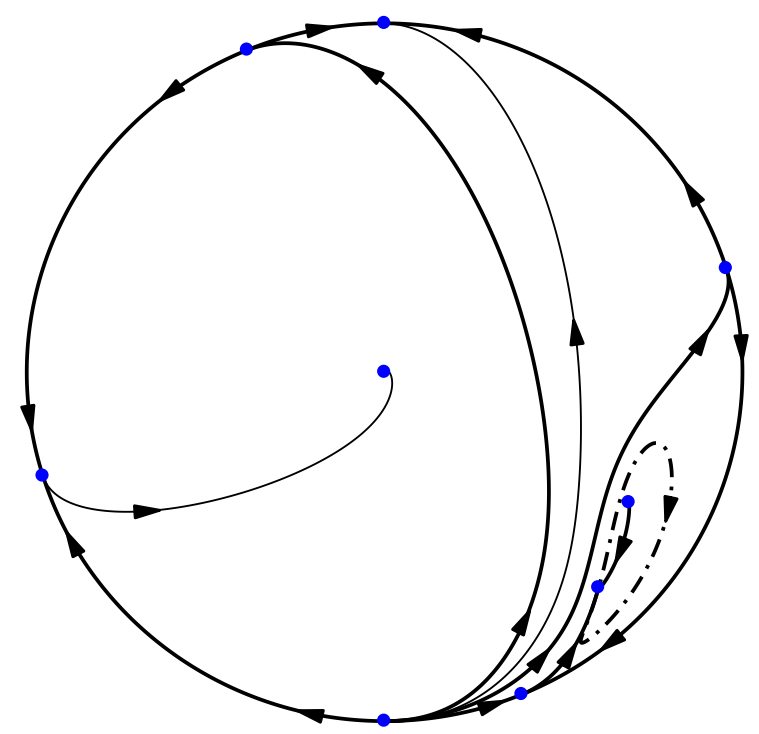

Figure 3. The phase portrait of system (7) when $c=\sqrt{c^{*}}$. It has 4 canonical regions and 21 separatrices. The dashdot line represents the homoclinic loop.

Then one eigenvalue is zero and the other one is nonzero. If $c^{2}>c^{*}$, in system (7) two singular points

$$
\begin{aligned}
E_{2}^{ \pm}= & \left(\frac{(a+b)\left(2 c^{2}+1\right) \pm|c| S_{a}}{4\left(c^{2}+1\right)},\right. \\
& \left.\quad-\frac{\left(8\left(a^{2}+b^{2}\right)\left(c^{2}+1\right)-3(a+b)^{2}\right) c^{2} \pm\left(4 c^{2}+1\right)(a+b)|c| S_{a}}{16 c\left(c^{2}+1\right)}\right)
\end{aligned}
$$

are bifurcated from $E_{2}$, where

$$
S_{a}=\sqrt{4(a-b)^{2} c^{2}+(3 a-b)(a-3 b)} .
$$

The characteristic equations at $E_{2}^{ \pm}$are in the form

$$
\lambda^{2}+b_{1}^{ \pm} \lambda+b_{0}^{ \pm}=0
$$


where

$$
\begin{gathered}
b_{1}^{ \pm}=\frac{c^{2}\left(12(a-b)^{2} c^{2}+11 a^{2}-26 a b+11 b^{2}\right) \pm(a+b)\left(6 c^{2}+5\right)|c| S_{a}}{4\left(1+c^{2}\right) c} \\
b_{0}^{ \pm}=\frac{\left((a+b)^{2}+4\left(a^{2}+b^{2}\right) c^{2}\right) S_{a}^{2}}{4\left(1+c^{2}\right)} \\
\pm \frac{(a+b)\left(8(a-b)^{2} c^{2}+\left(5 a^{2}-14 a b+5 b^{2}\right)\right)|c| S_{a}}{4\left(1+c^{2}\right)}
\end{gathered}
$$

Then their corresponding discriminants $\Delta^{ \pm}=b_{1}^{ \pm 2}-4 b_{0}^{ \pm}$are positive when $S_{a}=0$, i.e $c^{2}=c^{*}$. Hence equations (12) have real roots when $0<S_{a} \ll 1$. Note that $b_{0}^{ \pm}$have different signs for surfficiently small $S_{a}>0$. So the roots of one of these two equations have the same sign, and the other one's have different signs. Therefore, one of the singular points $E_{2}^{ \pm}$is a node point and the other one is a saddle point for $0<c^{2}-c^{*} \ll 1$. By long and tedious computations, $E_{2}^{ \pm}$are both also on the oval $\Gamma_{c}$.

\section{ACKNOWLEDGEMENTS}

The first author is partially supported by a MINECO-FEDER grant MTM2016-77278-P, a MINECO grant MTM2013-40998-P, and an AGAUR grant number 2014SGR-568. The second author is partially supported by the NNSF of China grant number 11501370 .

\section{REFERENCES}

[1] J. Chavarriga, H. Giacomini and J. Llibre, Uniqueness of algebraic limit cycles for quadratic systems, J. Math. Anal. and Appl. 261 (2001), 85-99.

[2] J. Chavarriga, J. Llibre and J. Moulin Ollagnier, On a result of Darboux, LMS J. of Computation and Mathematics 4 (2001), 197-210.

[3] J. Chavarriga, J. Llibre and J. Sorolla, Algebraic limit cycles of degree 4 for quadratic systems, J. Differential Equations 200 (2004), 206-244.

[4] C. Christopher and Chengzhi Li, Limit cycles of differential equations, Advanced Courses in Mathematics. CRM Barcelona. Birkhäuser Verlag, Basel, 2007.

[5] C. Christopher, J. Llibre and G. Świrszcz, Invariant algebraic curves of large degree for quadratic systems, J. Math. Anal. Appl., 303 (2005), 450-461.

[6] C. Chicone and J. Tian, On general properties of quadratic systems, The Amer. Math. Monthly 89 (1982), 167-178.

[7] W. A. Coppel, A survey of quadratic systems, J. Differential Equations 2 (1996), 293-304.

[8] F. Dumortier, J. Llibre and J.C. Artés, Qualitative theory of planar differential systems, UniversiText, Springer-Verlag, New York, 2006. 
[9] R.M. Evdokimenco, Construction of algebraic paths and the qualitative investigation in the large of the properties of integral curves of a system of differential equations, Differential Equations 6 (1970), 1349-1358.

[10] R.M. Evdokimenco, Behavior of integral curves of a dynamic system, Differential Equations 9 (1974), 1095-1103.

[11] R.M. Evdokimenco, Investigation in the large of a dynamic system, Differential Equations 15 (1979), 215-221.

[12] V.F. Filiptsov, Algebraic limit cycles, Differential Equations 9 (1973), 983986.

[13] J. Llibre And D. Schlomiuk, On the limit cycles bifurcating from an ellipse of a quadratic center, Discrete and Continuous Dynamical Systems-Series A 35 (2015), 1091-1102.

[14] J. Llibre And G. Swirszcz, Relationships between limit cycles and algebraic invariant curves for quadratic systems, J. Differential Equations 229 (2006), $529-537$.

[15] J. Llibre AND G. SwirszCZ, Classification of quadratic systems admitting the existence of an algebraic limit cycle, Bull. des Sciences Mathemàtiques 131 (2007), 405-421.

[16] L. MARKus, Global structure of ordinary differential equations in the plane, Trans. Amer. Math. Soc. 76 (1954), 127-148.

[17] D. Neumann, Classification of continuous flows on 2-manifolds, Proc. Amer. Math. Soc. 48 (1975), 73-81.

[18] M. Peiхото, Dynamical systems, Proceeding of a Symposium held at the University of Bahia, 389-420, Acad. Press, New York, 1973.

[19] QIN YUAN-Xun, On the algebraic limit cycles of second degree of the differential equation $d y / d x=\sum_{0 \leq i+j \leq 2} a_{i j} x^{i} y^{j} / \sum_{0<i+j \leq 2} b_{i j} x^{i} y^{j}$, Acta Math. Sinica 8 (1958), 23-35.

[20] D. Schlomiuk, Algebraic and Geometric Aspects of the Theory of Polynomial Vector Fields, in Bifurcations and Periodic Orbits of Vector Fields, NATO ASI Series, Series C-Vol. 408, (1993), 229-467.

[21] D. Schlomiuk, J. Guckenheimer And R. Rand, Integrability of plane quadratic vector fields, Expositiones Mathematicae 8 (1990), 3-25.

[22] Y. TIAN AND P. YU, An explicit recursive formula for computing the normal form and center manifold of $n$-dimensional differential systems associated with Hopf bifurcation, Int. J. Bifurcation and Chaos 23, (2013) 1350104.

[23] A.I. Yablonski, Limit cycles of a certain differential equations, Differential Equations 2 (1966), 335-344 (In Russian).

[24] Ye Yanqian, Theory of Limit Cycles, Translations of Math. Monographs, Vol. 66, Amer. Math. Soc, Providence, 1986.

${ }^{1}$ Departament de Matemàtiques, Universitat Autònoma de Barcelona, 08193 Bellaterra, Barcelona, Catalonia, Spain

E-mail address: jllibre@mat.uab.cat

2 Department of Mathematics, Shanghai Normal University, 100 Guilin Roda, Xuhui District, 200234, Shanghai, China

E-mail address: ytian22@shnu.edu.cn 